Supporting Information

\title{
Evaluation of 3-Carboxy-4-(1H)-Quinolones as Inhibitors of Human Protein Kinase CK2
}

Andriy G. Golub, Olexander Ya. Yakovenko, Volodymyr G. Bdzhola, Vladislav M. Sapelkin, Piotr Zien and Sergiy M. Yarmoluk

\section{Contents:}

Tables of atom-atom contacts:

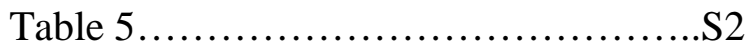

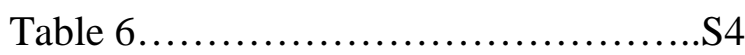

Table $7 \ldots \ldots \ldots \ldots \ldots \ldots \ldots \ldots \ldots \ldots \ldots . . . . . . . \ldots 6$

Spectroscopic Data.....................S8

Table of Elemental Analyses..............S12 
Table 5. Atom-atom contacts between inhibitor 7 and amino acid residues of the CK2 active site at distance cut-off $4.4 \AA$.

\begin{tabular}{|c|c|c|c|c|c|c|}
\hline \# & Ligand & Atom & & sidue & Atom & Distance \\
\hline 1 & 7 & C50 & $\operatorname{Trp}$ & 176 & CD1 & 4.3149 \\
\hline 2 & 7 & C50 & Asp & 175 & CA & 3.7324 \\
\hline 3 & 7 & $\mathrm{C} 00$ & Asp & 175 & CA & 4.0928 \\
\hline 4 & 7 & $\mathrm{C} 10$ & Asp & 175 & CA & 4.1049 \\
\hline 5 & 7 & $\mathrm{C} 20$ & Ile & 174 & $\mathrm{CD}$ & 3.8378 \\
\hline 6 & 7 & C60 & Ile & 174 & CD & 4.0257 \\
\hline 7 & 7 & C80 & Ile & 174 & CD & 4.2283 \\
\hline 8 & 7 & C70 & Ile & 174 & $\mathrm{CD}$ & 4.2263 \\
\hline 9 & 7 & $\mathrm{C} 40$ & Ile & 174 & $\mathrm{CD}$ & 4.0125 \\
\hline 10 & 7 & C & Ile & 174 & CD & 3.8114 \\
\hline 11 & 7 & C10 & Ile & 174 & CD & 4.2562 \\
\hline 12 & 7 & $\mathrm{C} 00$ & Ile & 174 & CG2 & 4.3262 \\
\hline 13 & 7 & C30 & Ile & 174 & CG2 & 3.4635 \\
\hline 14 & 7 & $\mathrm{C} 20$ & Ile & 174 & CG2 & 3.8765 \\
\hline 15 & 7 & CL0 & Ile & 174 & CG2 & 4.1054 \\
\hline 16 & 7 & C60 & Ile & 174 & CG2 & 4.2396 \\
\hline 17 & 7 & $\mathrm{COO}$ & Ile & 174 & CB & 3.9962 \\
\hline 18 & 7 & C30 & Ile & 174 & $\mathrm{CB}$ & 3.6245 \\
\hline 19 & 7 & $\mathrm{C} 20$ & Ile & 174 & CB & 4.0079 \\
\hline 20 & 7 & C10 & Ile & 174 & CB & 4.3634 \\
\hline 21 & 7 & $\mathrm{C} 00$ & Ile & 174 & $\mathrm{CA}$ & 4.3087 \\
\hline 22 & 7 & C30 & Ile & 174 & CA & 4.1231 \\
\hline 23 & 7 & CL0 & Met & 163 & $\mathrm{CE}$ & 4.2478 \\
\hline 24 & 7 & C80 & Met & 163 & $\mathrm{CE}$ & 4.2751 \\
\hline 25 & 7 & CLO & Met & 163 & SD & 3.617 \\
\hline 26 & 7 & C60 & Met & 163 & SD & 4.2433 \\
\hline 27 & 7 & CL0 & Val & 116 & CG1 & 3.7187 \\
\hline 28 & 7 & C50 & Phe & 113 & $\mathrm{CZ}$ & 3.5775 \\
\hline 29 & 7 & $\mathrm{C} 00$ & Phe & 113 & $\mathrm{CZ}$ & 3.6837 \\
\hline 30 & 7 & C30 & Phe & 113 & $\mathrm{CZ}$ & 3.6514 \\
\hline 31 & 7 & C50 & Phe & 113 & CE2 & 4.106 \\
\hline 32 & 7 & $\mathrm{C} 00$ & Phe & 113 & CE2 & 3.7415 \\
\hline 33 & 7 & C30 & Phe & 113 & CE2 & 3.7182 \\
\hline 34 & 7 & C10 & Phe & 113 & CE2 & 4.1773 \\
\hline 35 & 7 & C50 & Phe & 113 & CE1 & 4.0754 \\
\hline 36 & 7 & $\mathrm{C} 00$ & Phe & 113 & CE1 & 4.1984 \\
\hline 37 & 7 & C30 & Phe & 113 & CE1 & 3.7538 \\
\hline 38 & 7 & $\mathrm{C} 00$ & Phe & 113 & CD2 & 4.1951 \\
\hline 39 & 7 & C30 & Phe & 113 & CD2 & 3.7532 \\
\hline 40 & 7 & $\mathrm{C} 20$ & Phe & 113 & CD2 & 4.1793 \\
\hline 41 & 7 & C30 & Phe & 113 & CD1 & 3.8152 \\
\hline 42 & 7 & C30 & Phe & 113 & CG & 3.8364 \\
\hline 43 & 7 & C50 & Ile & 95 & CG2 & 4.2645 \\
\hline 44 & 7 & C30 & Ile & 95 & CG2 & 3.6297 \\
\hline 45 & 7 & C30 & Ile & 95 & CG1 & 3.5426 \\
\hline
\end{tabular}




\begin{tabular}{|l|l|l|l|l|l|l|}
\hline $\mathbf{4 6}$ & $\mathbf{7}$ & C30 & Ile & 95 & CB & 4.0755 \\
\hline $\mathbf{4 7}$ & $\mathbf{7}$ & C50 & Leu & 85 & CD1 & 4.1491 \\
\hline $\mathbf{4 8}$ & $\mathbf{7}$ & C50 & Glu & 81 & CD & 4.2534 \\
\hline $\mathbf{4 9}$ & $\mathbf{7}$ & CL & Lys & 68 & CE & 4.0706 \\
\hline $\mathbf{5 0}$ & $\mathbf{7}$ & C10 & Lys & 68 & CD & 4.2815 \\
\hline $\mathbf{5 1}$ & $\mathbf{7}$ & CL0 & Val & 66 & CG2 & 4.2907 \\
\hline $\mathbf{5 2}$ & $\mathbf{7}$ & CL0 & Val & 66 & CG1 & 3.9635 \\
\hline $\mathbf{5 3}$ & $\mathbf{7}$ & C60 & Val & 66 & CG1 & 3.8703 \\
\hline $\mathbf{5 4}$ & $\mathbf{7}$ & C80 & Val & 66 & CG1 & 3.8339 \\
\hline $\mathbf{5 5}$ & $\mathbf{7}$ & CL0 & Val & 66 & CB & 4.1486 \\
\hline
\end{tabular}


Table 6. Atom-atom contacts between inhibitor 9 and amino acid residues of the CK2 active site at distance cut-off $4.4 \AA$.

\begin{tabular}{|c|c|c|c|c|c|c|}
\hline \# & Ligand & Atom & & sidue & Atom & Distance \\
\hline 1 & 9 & $\mathrm{C} 40$ & Asp & 175 & CG & 3.38 \\
\hline 2 & 9 & C & Asp & 175 & CG & 4.4 \\
\hline 3 & 9 & C40 & Asp & 175 & CB & 4.29 \\
\hline 4 & 9 & C40 & Asp & 175 & CA & 4.22 \\
\hline 5 & 9 & $\mathrm{C} 40$ & Ile & 174 & $\mathrm{CD}$ & 3.82 \\
\hline 6 & 9 & C & Ile & 174 & $\mathrm{CD}$ & 3.56 \\
\hline 7 & 9 & C30 & Ile & 174 & $\mathrm{CD}$ & 4.1 \\
\hline 8 & 9 & C10 & Ile & 174 & $\mathrm{CD}$ & 4.17 \\
\hline 9 & 9 & $\mathrm{COO}$ & Ile & 174 & CD & 3.6 \\
\hline 10 & 9 & C & Ile & 174 & CG2 & 4.25 \\
\hline 11 & 9 & C30 & Ile & 174 & CG2 & 3.66 \\
\hline 12 & 9 & $\mathrm{C}$ & Ile & 174 & CG1 & 4.28 \\
\hline 13 & 9 & C30 & Ile & 174 & CG1 & 4.39 \\
\hline 14 & 9 & $\mathrm{C} 40$ & Ile & 174 & CB & 3.99 \\
\hline 15 & 9 & $\mathrm{C}$ & Ile & 174 & CB & 4 \\
\hline 16 & 9 & C30 & Ile & 174 & CB & 3.91 \\
\hline 17 & 9 & C70 & Met & 163 & $\mathrm{CE}$ & 3.96 \\
\hline 18 & 9 & C80 & Met & 163 & $\mathrm{CE}$ & 3.5 \\
\hline 19 & 9 & C60 & Met & 163 & $\mathrm{CE}$ & 3.64 \\
\hline 20 & 9 & C10 & Met & 163 & $\mathrm{CE}$ & 4.19 \\
\hline 21 & 9 & C20 & Met & 163 & CG & 4.27 \\
\hline 22 & 9 & C50 & Met & 163 & CG & 4.13 \\
\hline 23 & 9 & C70 & Met & 163 & CG & 4.4 \\
\hline 24 & 9 & CA0 & Thr & 119 & CG2 & 4.03 \\
\hline 25 & 9 & CA0 & Asn & 118 & C & 4.19 \\
\hline 26 & 9 & CB0 & Val & 116 & C & 4.17 \\
\hline 27 & 9 & C90 & Val & 116 & CG1 & 3.92 \\
\hline 28 & 9 & CB0 & Val & 116 & CG1 & 4.11 \\
\hline 29 & 9 & C30 & Phe & 113 & CE2 & 4.25 \\
\hline 30 & 9 & $\mathrm{C} 40$ & Lys & 68 & $\mathrm{CE}$ & 4.22 \\
\hline 31 & 9 & C40 & Lys & 68 & $\mathrm{CD}$ & 4.05 \\
\hline 32 & 9 & C90 & Val & 66 & CG2 & 4.28 \\
\hline 33 & 9 & CB0 & Val & 66 & CG2 & 4.35 \\
\hline 34 & 9 & C30 & Val & 66 & CG1 & 4.29 \\
\hline 35 & 9 & $\mathrm{C} 20$ & Val & 66 & CG1 & 4.38 \\
\hline 36 & 9 & C90 & Val & 66 & CG1 & 3.85 \\
\hline 37 & 9 & C40 & Val & 53 & CG2 & 4.06 \\
\hline 38 & 9 & C & Val & 53 & CG2 & 3.55 \\
\hline 39 & 9 & C30 & Val & 53 & CG2 & 4.14 \\
\hline 40 & 9 & C20 & Val & 53 & CG2 & 4.23 \\
\hline 41 & 9 & C60 & Val & 53 & CG2 & 4.19 \\
\hline 42 & 9 & C10 & Val & 53 & CG2 & 3.64 \\
\hline 43 & 9 & $\mathrm{COO}$ & Val & 53 & CG2 & 3.23 \\
\hline 44 & 9 & C20 & Val & 53 & CG1 & 4.11 \\
\hline 45 & 9 & C10 & Val & 53 & CG1 & 4.27 \\
\hline
\end{tabular}




\begin{tabular}{|l|l|l|l|l|l|l|}
\hline $\mathbf{4 6}$ & $\mathbf{9}$ & C10 & Val & 53 & CB & 4.2 \\
\hline $\mathbf{4 7}$ & $\mathbf{9}$ & C00 & Val & 53 & CB & 4.26 \\
\hline $\mathbf{4 8}$ & $\mathbf{9}$ & C60 & Gly & 46 & CA & 4.14 \\
\hline $\mathbf{4 9}$ & $\mathbf{9}$ & C70 & Leu & 45 & C & 4.19 \\
\hline $\mathbf{5 0}$ & $\mathbf{9}$ & C80 & Leu & 45 & C & 3.47 \\
\hline $\mathbf{5 1}$ & $\mathbf{9}$ & C60 & Leu & 45 & C & 3.82 \\
\hline $\mathbf{5 2}$ & $\mathbf{9}$ & CC0 & Leu & 45 & CD2 & 3.97 \\
\hline $\mathbf{5 3}$ & $\mathbf{9}$ & CA0 & Leu & 45 & CD2 & 4.15 \\
\hline $\mathbf{5 4}$ & $\mathbf{9}$ & C50 & Leu & 45 & CD1 & 4.4 \\
\hline $\mathbf{5 5}$ & $\mathbf{9}$ & C90 & Leu & 45 & CD1 & 4.12 \\
\hline $\mathbf{5 6}$ & $\mathbf{9}$ & CB0 & Leu & 45 & CD1 & 3.9 \\
\hline $\mathbf{5 7}$ & $\mathbf{9}$ & CC0 & Leu & 45 & CD1 & 4.04 \\
\hline $\mathbf{5 8}$ & $\mathbf{9}$ & CA0 & Leu & 45 & CD1 & 4.36 \\
\hline $\mathbf{5 9}$ & $\mathbf{9}$ & CC0 & Leu & 45 & CG & 4.25 \\
\hline $\mathbf{6 0}$ & $\mathbf{9}$ & CA0 & Leu & 45 & CG & 4.24 \\
\hline $\mathbf{6 1}$ & $\mathbf{9}$ & C50 & Leu & 45 & CB & 4.06 \\
\hline $\mathbf{6 2}$ & $\mathbf{9}$ & CC0 & Leu & 45 & CB & 4.04 \\
\hline $\mathbf{6 3}$ & $\mathbf{9}$ & CA0 & Leu & 45 & CB & 3.56 \\
\hline $\mathbf{6 4}$ & $\mathbf{9}$ & C70 & Leu & 45 & CB & 3.54 \\
\hline $\mathbf{6 5}$ & $\mathbf{9}$ & C80 & Leu & 45 & CB & 3.72 \\
\hline $\mathbf{6 6}$ & $\mathbf{9}$ & C60 & Leu & 45 & CB & 4.34 \\
\hline $\mathbf{6 7}$ & $\mathbf{9}$ & CA0 & Leu & 45 & CA & 4.19 \\
\hline $\mathbf{6 8}$ & $\mathbf{9}$ & C70 & Leu & 45 & CA & 4.27 \\
\hline $\mathbf{6 9}$ & $\mathbf{9}$ & C80 & Leu & 45 & CA & 4.03 \\
\hline
\end{tabular}


Table 7. Atom-atom contacts between IQA and amino acid residues of the CK2 active site at distance cut-off $4.4 \AA$.

\begin{tabular}{|c|c|c|c|c|c|c|}
\hline$\#$ & Ligand & Atom & \multicolumn{2}{|c|}{ Residue } & Atom & Distance \\
\hline 1 & IQA & $\mathrm{C} 20$ & Asp & 175 & $\mathrm{CG}$ & 3.96 \\
\hline 2 & IQA & $\mathrm{C} 20$ & Asp & 175 & $\mathrm{CB}$ & 3.64 \\
\hline 3 & IQA & $\mathrm{C} 20$ & Asp & 175 & $\mathrm{CA}$ & 3.43 \\
\hline 4 & IQA & C6 & Ile & 174 & CD1 & 4.1 \\
\hline 5 & IQA & $\mathrm{C} 8$ & Ile & 174 & CD1 & 3.5 \\
\hline 6 & IQA & C5 & Ile & 174 & CD1 & 3.49 \\
\hline 7 & IQA & C18 & Ile & 174 & CD1 & 3.74 \\
\hline 8 & IQA & $\mathrm{C} 20$ & Ile & 174 & CD1 & 4.37 \\
\hline 9 & IQA & $\mathrm{C} 1$ & Ile & 174 & CG2 & 4.14 \\
\hline 10 & IQA & C4 & Ile & 174 & CG2 & 3.83 \\
\hline 11 & IQA & C6 & Ile & 174 & $\mathrm{CG} 2$ & 3.89 \\
\hline 12 & IQA & C7 & Ile & 174 & CG2 & 4.25 \\
\hline 13 & IQA & C5 & Ile & 174 & CG1 & 4.39 \\
\hline 14 & IQA & $\mathrm{C} 4$ & Ile & 174 & $\mathrm{CB}$ & 4.13 \\
\hline 15 & IQA & C6 & Ile & 174 & CB & 4.1 \\
\hline 16 & IQA & C5 & Ile & 174 & CB & 4.17 \\
\hline 17 & IQA & C18 & Ile & 174 & CB & 4.37 \\
\hline 18 & IQA & $\mathrm{C} 20$ & Ile & 174 & CB & 4.26 \\
\hline 19 & IQA & C13 & Met & 163 & SD & 3.58 \\
\hline 20 & IQA & C14 & Met & 163 & SD & 3.79 \\
\hline 21 & IQA & C15 & Met & 163 & SD & 4.21 \\
\hline 22 & IQA & C17 & Met & 163 & SD & 4.23 \\
\hline 23 & IQA & C12 & Met & 163 & SD & 3.82 \\
\hline 24 & IQA & C13 & Met & 163 & $\mathrm{CG}$ & 4.4 \\
\hline 25 & IQA & C14 & Asn & 118 & $\mathrm{CB}$ & 4.35 \\
\hline 26 & IQA & C15 & Asn & 118 & $\mathrm{CB}$ & 3.7 \\
\hline 27 & IQA & C16 & Asn & 118 & CB & 4.07 \\
\hline 28 & $\begin{array}{l}\text { IQA } \\
\end{array}$ & C3 & Val & 116 & CG2 & 4.11 \\
\hline 29 & IQA & C2 & Val & 116 & CG2 & 3.53 \\
\hline 30 & IQA & C3 & Val & 116 & CB & 3.71 \\
\hline 31 & IQA & C2 & Val & 116 & CB & 3.69 \\
\hline 32 & IQA & C4 & Phe & 113 & CE2 & 4.15 \\
\hline 33 & IQA & C1 & Phe & 113 & CD2 & 3.92 \\
\hline 34 & IQA & $\mathrm{C} 4$ & Phe & 113 & CD2 & 3.78 \\
\hline 35 & IQA & C1 & Phe & 113 & CD1 & 4.27 \\
\hline 36 & IQA & C1 & Phe & 113 & $\mathrm{CG}$ & 3.68 \\
\hline 37 & IQA & C4 & Phe & 113 & $\mathrm{CG}$ & 3.99 \\
\hline 38 & IQA & $\mathrm{C} 2$ & Phe & 113 & CB & 4.2 \\
\hline 39 & $\begin{array}{l}\text { IQA } \\
\end{array}$ & C1 & Phe & 113 & CB & 3.53 \\
\hline 40 & IQA & C4 & Phe & 113 & CB & 4.31 \\
\hline 41 & IQA & C2 & Val & 95 & CG2 & 4.08 \\
\hline 42 & IQA & C1 & Val & 95 & CG2 & 3.16 \\
\hline 43 & IQA & $\mathrm{C} 4$ & Val & 95 & CG2 & 3.94 \\
\hline 44 & IQA & C1 & Val & 95 & $\mathrm{CB}$ & 4.05 \\
\hline 45 & IQA & C20 & Lys & 68 & $\mathrm{CE}$ & 3.83 \\
\hline
\end{tabular}




\begin{tabular}{|c|c|c|c|c|c|c|}
\hline 46 & IQA & C18 & Lys & 68 & $\mathrm{CD}$ & 4.35 \\
\hline 47 & IQA & $\mathrm{C} 20$ & Lys & 68 & $\mathrm{CD}$ & 3.64 \\
\hline 48 & IQA & C3 & Ile & 66 & CD1 & 3.9 \\
\hline 49 & IQA & $\mathrm{C} 14$ & Ile & 66 & CD1 & 4.09 \\
\hline 50 & IQA & C3 & Ile & 66 & CG2 & 3.82 \\
\hline 51 & IQA & $\mathrm{C} 2$ & Ile & 66 & CG2 & 4.01 \\
\hline 52 & IQA & $\mathrm{C} 1$ & Ile & 66 & CG2 & 4.17 \\
\hline 53 & IQA & C4 & Ile & 66 & CG2 & 4.14 \\
\hline 54 & IQA & C6 & Ile & 66 & CG2 & 3.94 \\
\hline 55 & IQA & C7 & Ile & 66 & CG2 & 3.78 \\
\hline 56 & IQA & C3 & Ile & 66 & CG1 & 3.38 \\
\hline 57 & IQA & $\mathrm{C} 2$ & Ile & 66 & CG1 & 3.57 \\
\hline 58 & IQA & C7 & Ile & 66 & CG1 & 4.17 \\
\hline 59 & IQA & C3 & Ile & 66 & CB & 3.94 \\
\hline 60 & IQA & $\mathrm{C} 2$ & Ile & 66 & CB & 3.85 \\
\hline 61 & IQA & $\mathrm{C} 1$ & Ile & 66 & CB & 4.26 \\
\hline 62 & IQA & $\mathrm{C} 10$ & Val & 53 & CG2 & 4.22 \\
\hline 63 & IQA & C8 & Val & 53 & CG2 & 4.04 \\
\hline 64 & IQA & C5 & Val & 53 & CG2 & 4.2 \\
\hline 65 & IQA & C18 & Val & 53 & CG2 & 3.89 \\
\hline 66 & IQA & $\mathrm{C} 13$ & Val & 53 & CG1 & 4.26 \\
\hline 67 & IQA & C12 & Val & 53 & CG1 & 4.33 \\
\hline 68 & IQA & $\mathrm{C} 10$ & Val & 53 & CG1 & 4.09 \\
\hline 69 & IQA & C8 & Val & 53 & CG1 & 3.71 \\
\hline 70 & IQA & C5 & Val & 53 & CG1 & 4.14 \\
\hline 71 & IQA & $\mathrm{C} 10$ & Val & 53 & CB & 4.09 \\
\hline 72 & IQA & C8 & Val & 53 & CB & 4.27 \\
\hline 73 & IQA & C17 & Gly & 46 & CA & 4.4 \\
\hline 74 & IQA & $\mathrm{C} 10$ & Gly & 46 & CA & 4.2 \\
\hline 75 & IQA & $\mathrm{C} 13$ & Val & 45 & CG2 & 4.19 \\
\hline 76 & IQA & C14 & Val & 45 & CG2 & 4.22 \\
\hline 77 & IQA & C15 & Val & 45 & CG2 & 4.06 \\
\hline 78 & IQA & C16 & Val & 45 & CG2 & 3.85 \\
\hline 79 & IQA & C17 & Val & 45 & CG2 & 3.8 \\
\hline 80 & IQA & C12 & Val & 45 & CG2 & 3.97 \\
\hline 81 & IQA & C15 & Val & 45 & CB & 4.16 \\
\hline 82 & IQA & C16 & Val & 45 & CB & 3.7 \\
\hline 83 & IQA & C17 & Val & 45 & CB & 3.96 \\
\hline 84 & IQA & C16 & Val & 45 & C & 4.25 \\
\hline 85 & IQA & C17 & Val & 45 & C & 3.79 \\
\hline
\end{tabular}


Spectroscopic Data:

6-Iodoquinoline-4-one-3-carboxylic acid 1

Mp 289-291 ${ }^{\circ} \mathrm{C}:{ }^{1} \mathrm{H}$ NMR (DMSO-d $\left.{ }_{6}\right) \delta 14,83$ (brs, $\left.1 \mathrm{H}\right), 13,34$ (brs, $\left.1 \mathrm{H}\right), 8,85$ (s,1H), 8,53 (s,1H), 8,11 $(\mathrm{d}, 1 \mathrm{H}, \mathrm{J}=8,1 \mathrm{~Hz}), 7,6(\mathrm{~d}, 1 \mathrm{H}, \mathrm{J}=8,1 \mathrm{~Hz})$. Anal. $\left(\mathrm{C}_{10} \mathrm{H}_{6} \mathrm{INO}_{3}\right) \mathrm{C}, \mathrm{H}, \mathrm{N}, \mathrm{I}$

Quinoline-4-one-3,6-dicarboxylic acid 2

$\mathrm{Mp}>300{ }^{\circ} \mathrm{C}:{ }^{1} \mathrm{H}$ NMR $\left(\mathrm{DMSO}_{6}\right) \delta 14,08$ (brs, $\left.1 \mathrm{H}\right), 13,95$ (brs, $\left.1 \mathrm{H}\right), 13,36$ (brs, $\left.1 \mathrm{H}\right), 8,91$ (s, 1H), 8,83 $(\mathrm{d}, 1 \mathrm{H}, \mathrm{J}=1,9 \mathrm{~Hz}), 8,34(\mathrm{dd}, 1 \mathrm{H}, \mathrm{J}=8,7 \mathrm{~Hz}, \mathrm{~J}=1,9 \mathrm{~Hz}), 7,95(\mathrm{~d}, 1 \mathrm{H} \mathrm{J}=8,7 \mathrm{~Hz})$. Anal. $\left(\mathrm{C}_{11} \mathrm{H}_{7} \mathrm{NO}_{5}\right) \mathrm{C}, \mathrm{H}, \mathrm{N}$

Quinoline-4-one-3,7-dicarboxylic acid 3

Mp >300 ${ }^{\circ} \mathrm{C}:{ }^{1} \mathrm{H}$ NMR (DMSO-d 6 ) $\delta$ 14,75 (brs,1H), 13,61 (brs,2H), 8,98 (s,1H), 8,37 (s,1H), 8,35 $(\mathrm{d}, 1 \mathrm{H}, \mathrm{J}=8,4 \mathrm{~Hz}), 8,03(\mathrm{~d}, 1 \mathrm{H}, \mathrm{J}=8,4 \mathrm{~Hz})$. Anal. $\left(\mathrm{C}_{11} \mathrm{H}_{7} \mathrm{NO}_{5}\right) \mathrm{C}, \mathrm{H}, \mathrm{N}$

Quinoline-4-one-3,8-dicarboxylic acid 4

Mp >300(295-297) ${ }^{\circ} \mathrm{C}:{ }^{1} \mathrm{H}$ NMR (DMSO-d $)_{6} \delta$ 14,54 (brs,1H), 13,09 (d,1H,J=6,2 Hz), 8,94 $(\mathrm{d}, 1 \mathrm{H}, \mathrm{J}=6,8 \mathrm{~Hz}), 8,52(\mathrm{~d}, 2 \mathrm{H}, \mathrm{J}=7,8 \mathrm{~Hz}), 7,68(\mathrm{t}, 1 \mathrm{H}, \mathrm{J}=7,8 \mathrm{~Hz})$. Anal. $\left(\mathrm{C}_{11} \mathrm{H}_{7} \mathrm{NO}_{5}\right) \mathrm{C}, \mathrm{H}, \mathrm{N}$

6,7-Etylenedioxy-quinoline-4-one-3-carboxylic acid $\mathbf{5}$

Mp 277-279 ${ }^{\circ} \mathrm{C}:{ }^{1} \mathrm{H}$ NMR (DMSO-d $\left.{ }_{6}\right) \delta$ 13,54 (brs,1H), 8,68 (d,1H,J=6,2Hz), 7,56 (s,1H), 7,29 $(\mathrm{s}, 1 \mathrm{H}), 4,40(\mathrm{~m}, 4 \mathrm{H})$. Anal. $\left(\mathrm{C}_{12} \mathrm{H}_{9} \mathrm{NO}_{5}\right) \mathrm{C}, \mathrm{H}, \mathrm{N}$

6,8-Dichloroquinoline-4-one-3-carboxylic acid 6

$\mathrm{Mp}>300{ }^{\circ} \mathrm{C}:{ }^{1} \mathrm{H}$ NMR $\left(\mathrm{DMSO}_{6}\right) \delta 14,51$ (brs, $\left.1 \mathrm{H}\right), 13,14$ (brs, $\left.1 \mathrm{H}\right), 8,66(\mathrm{~s}, 1 \mathrm{H}), 8,24(\mathrm{~d}, 1 \mathrm{H}, \mathrm{J}=2,4$ $\mathrm{Hz}), 8,16(\mathrm{~d}, 1 \mathrm{H}, \mathrm{J}=2,4 \mathrm{~Hz})$. Anal. $\left(\mathrm{C}_{10} \mathrm{H}_{5} \mathrm{Cl}_{2} \mathrm{NO}_{3}\right) \mathrm{C}, \mathrm{H}, \mathrm{N}, \mathrm{Cl}$

5,6,8-Trichloroquinoline-4-one-3-carboxylic acid 7

Mp - ${ }^{\circ} \mathrm{C}:{ }^{1} \mathrm{H}$ NMR (DMSO-d 6 ) $\delta$ 14,60 (brs, $\left.1 \mathrm{H}\right), 12,75$ (brs,1H), 8,57 (s,1H), 8,37 (s,1H). Anal. $\left(\mathrm{C}_{10} \mathrm{H}_{4} \mathrm{Cl}_{3} \mathrm{NO}_{3}\right) \mathrm{C}, \mathrm{H}, \mathrm{N}, \mathrm{Cl}$

3-methyl-9,10-difluoro -2,3-dihydro-7-oxo-7H-1,4-oxazino[2,3,4-ij]quinoline-6-carboxylic acid 8

Mp 298-300 ${ }^{\circ} \mathrm{C}:{ }^{1} \mathrm{H}$ NMR (DMSO-d 6$) \delta 14,67$ (brs, 1H), 9,04 (s,1H), 7,79 (t,1H), 5,02 (m,1H),4,72 $(\mathrm{dd}, 2 \mathrm{H}), 1,51(\mathrm{~d}, 1 \mathrm{H})$. Anal. $\left(\mathrm{C}_{13} \mathrm{H}_{9} \mathrm{~F}_{2} \mathrm{NO}_{4}\right) \mathrm{C}, \mathrm{H}, \mathrm{N}$

1,4-dihydro-4-oxo-benzo[ $h]$ quinoline-3-carboxylic acid 9

$\mathrm{Mp}>300{ }^{\circ} \mathrm{C}:{ }^{1} \mathrm{H}$ NMR $\left(\mathrm{DMSO}_{6} \mathrm{~d}_{6}\right) \delta 15,51$ (brs, $\left.1 \mathrm{H}\right), 13,96$ (brs, $\left.1 \mathrm{H}\right), 8,89(\mathrm{~m}, 1 \mathrm{H}), 8,73(\mathrm{~s}, 1 \mathrm{H}), 8,15$ $(\mathrm{d}, 1 \mathrm{H}, \mathrm{J}=8,7 \mathrm{~Hz}), 8,09(\mathrm{~m}, 1 \mathrm{H}), 7,92(\mathrm{~d}, 1 \mathrm{H}, \mathrm{J}=8,7 \mathrm{~Hz}), 7,83(\mathrm{~m}, 2 \mathrm{H})$ Anal. $\left(\mathrm{C}_{14} \mathrm{H}_{9} \mathrm{NO}_{3}\right) \mathrm{C}, \mathrm{H}, \mathrm{N}$

6-Ethoxyquinoline-4-one-3-carboxylic acid $\mathbf{1 0}$

Mp 283-285 ${ }^{\circ} \mathrm{C}:{ }^{1} \mathrm{H}$ NMR (DMSO-d 6 ) $\delta 15,57$ (brs, $\left.1 \mathrm{H}\right), 13,77$ (brs, $\left.1 \mathrm{H}\right), 8,77(\mathrm{~s}, 1 \mathrm{H}), 7,83(\mathrm{~d}, 1 \mathrm{H}, \mathrm{J}=9,0$ $\mathrm{Hz}), 7,58(\mathrm{~s}, 1 \mathrm{H}), 7,50(\mathrm{dd}, 1 \mathrm{H}, \mathrm{J}=9,0 \mathrm{~Hz}, \mathrm{~J}=2,2 \mathrm{~Hz}), 4,17(\mathrm{q}, 2 \mathrm{H}), 1,41(\mathrm{t}, 3 \mathrm{H})$. Anal. $\left(\mathrm{C}_{12} \mathrm{H}_{11} \mathrm{NO}_{4}\right) \mathrm{C}, \mathrm{H}, \mathrm{N}$

6-Methoxyquinoline-4-one-3-carboxylic acid 11

Mp 279-280 ${ }^{\circ} \mathrm{C}:{ }^{1} \mathrm{H}$ NMR (DMSO-d 6 ) $\delta 15,47$ (brs, $\left.1 \mathrm{H}\right), 13,49$ (brs, $\left.1 \mathrm{H}\right), 8,76(\mathrm{~s}, 1 \mathrm{H}), 7,80(\mathrm{~d}, 1 \mathrm{H}, \mathrm{J}=9,0$ $\mathrm{Hz}), 7,63(\mathrm{~d}, 1 \mathrm{H}, \mathrm{J}=2,8 \mathrm{~Hz}), 7,50(\mathrm{dd}, 1 \mathrm{H}, \mathrm{J}=9,0 \mathrm{~Hz}, \mathrm{~J}=2,8 \mathrm{~Hz}), 3,91(\mathrm{~s}, 3 \mathrm{H})$. Anal. $\left(\mathrm{C}_{11} \mathrm{H}_{9} \mathrm{NO}_{4}\right) \mathrm{C}, \mathrm{H}, \mathrm{N}$

Quinoline-4-one-3-carboxylic acid 12

Mp 251-253 ${ }^{\circ} \mathrm{C}:{ }^{1} \mathrm{H}$ NMR (DMSO-d $) \delta 15,35$ (brs, $\left.1 \mathrm{H}\right), 13,46$ (brs, $\left.1 \mathrm{H}\right), 8,89(\mathrm{~s}, 1 \mathrm{H}), 8,30(\mathrm{~d}, 1 \mathrm{H}, \mathrm{J}=8,1$ $\mathrm{Hz}), 7,87(\mathrm{~m}, 2 \mathrm{H}), 7,61(\mathrm{dd}, 1, \mathrm{H} \mathrm{J}=7,5 \mathrm{~Hz}, \mathrm{~J}=7,8 \mathrm{~Hz})$. Anal. $\left(\mathrm{C}_{10} \mathrm{H}_{7} \mathrm{NO}_{3}\right) \mathrm{C}, \mathrm{H}, \mathrm{N}$ 
8-Ethoxyquinoline-4-one-3-carboxylic acid 13

Mp 269-270 ${ }^{\circ} \mathrm{C}:{ }^{1} \mathrm{H}$ NMR $\left(\mathrm{DMSO}_{6}\right) \delta 15,26$ (brs, $\left.1 \mathrm{H}\right), 12,90($ brd, $1 \mathrm{H}, \mathrm{J}=5,9 \mathrm{~Hz}), 8,60(\mathrm{~d}, 1 \mathrm{H}, \mathrm{J}=6,8$ $\mathrm{Hz}), 7,79(\mathrm{~d}, 1 \mathrm{H}, \mathrm{J}=7,8 \mathrm{~Hz}), 7,48(\mathrm{~m}, 2 \mathrm{H}), 4,31(\mathrm{q}, 2 \mathrm{H}), 1,50(\mathrm{t}, 3 \mathrm{H})$. Anal. $\left(\mathrm{C}_{12} \mathrm{H}_{11} \mathrm{NO}_{4}\right) \mathrm{C}, \mathrm{H}, \mathrm{N}$

3-methyl 9-fluoro -10-(4-methylpiperazin-1-yl) -2,3-dihydro-7-oxo-7H-[1,4]oxazino[2,3,4ij]quinoline-6-carboxylic acid 14

Mp 233-234 ${ }^{\circ} \mathrm{C}:{ }^{1} \mathrm{H}$ NMR (DMSO-d $\left.{ }_{6}\right) \delta 14,92$ (brs, $\left.1 \mathrm{H}\right), 8,88(\mathrm{~s}, 1 \mathrm{H}), 7,53$ (d,1H,J=12,3 Hz), 4,90 $(\mathrm{m}, 1 \mathrm{H}), 4,64(\mathrm{dd}, 2 \mathrm{H}), 2,24(\mathrm{~s}, 3 \mathrm{H}), 1,47(\mathrm{~d}, 3 \mathrm{H})$. Anal. $\left(\mathrm{C}_{18} \mathrm{H}_{20} \mathrm{FN}_{3} \mathrm{O}_{4}\right) \mathrm{C}, \mathrm{H}, \mathrm{N}$

6-Methylquinoline-4-one-3-carboxylic acid $\mathbf{1 5}$

Mp 280-281 ${ }^{\circ} \mathrm{C}:{ }^{1} \mathrm{H}$ NMR (DMSO-d $\left.{ }_{6}\right) \delta 15,46$ (brs, $\left.1 \mathrm{H}\right), 13,45$ (brs, $\left.1 \mathrm{H}\right), 8,82(\mathrm{~s}, 1 \mathrm{H}), 8,07(\mathrm{~s}, 1 \mathrm{H}), 7,73$ $(\mathrm{m}, 2 \mathrm{H}), 2,49(\mathrm{~s}, 3 \mathrm{H})$. Anal. $\left(\mathrm{C}_{11} \mathrm{H}_{9} \mathrm{NO}_{3}\right) \mathrm{C}, \mathrm{H}, \mathrm{N}$

6-Acetylquinoline-4-one-3-carboxylic acid $\mathbf{1 6}$

Mp 277-278 ${ }^{\circ} \mathrm{C}:{ }^{1} \mathrm{H}$ NMR (DMSO-d $) ~ \delta ~ 14,98$ (brs, $\left.1 \mathrm{H}\right), 13,73$ (brs, $\left.1 \mathrm{H}\right), 8,93$ (s,1H), 8,79 (d,1H,J=1,6 $\mathrm{Hz}), 8,34(\mathrm{dd}, 1 \mathrm{H}, \mathrm{J}=8,7 \mathrm{~Hz}, \mathrm{~J}=1,6 \mathrm{~Hz}), 7,91(\mathrm{~d}, 1 \mathrm{H}, \mathrm{J}=8,7 \mathrm{~Hz}), 2,71(\mathrm{~s}, 3 \mathrm{H})$. Anal. $\left(\mathrm{C}_{12} \mathrm{H}_{9} \mathrm{NO}_{4}\right) \mathrm{C}, \mathrm{H}, \mathrm{N}$

6,8-Dimethoxyquinoline-4-one-3-carboxylic acid $\mathbf{1 7}$

$\mathrm{Mp}>300{ }^{\circ} \mathrm{C}:{ }^{1} \mathrm{H}$ NMR $\left(\mathrm{DMSO}_{6}\right) \delta 15,55$ (brs, $\left.1 \mathrm{H}\right), 13,10$ (brs, $\left.1 \mathrm{H}\right), 8,50$ (d,1H,J=6,8 Hz), 7,21 $(\mathrm{d}, 1 \mathrm{H}, 1,9 \mathrm{~Hz}), 7,12(\mathrm{~d}, 1 \mathrm{H}, \mathrm{J}=2,5 \mathrm{~Hz}), 4,05(\mathrm{~s}, 3 \mathrm{H}), 3,91(\mathrm{~s}, 3 \mathrm{H})$. Anal. $\left(\mathrm{C}_{12} \mathrm{H}_{11} \mathrm{NO}_{5}\right) \mathrm{C}, \mathrm{H}, \mathrm{N}$

8-Isopropylquinoline-4-one-3-carboxylic acid $\mathbf{1 8}$

Mp 277-279 ${ }^{\circ} \mathrm{C}:{ }^{1} \mathrm{H}$ NMR (DMSO-d 6 ) $\delta$ 15,35 (brs,1H), 13,01 (brs,1H), 8,67 (s,1H), 8,12 $(\mathrm{dd}, 1 \mathrm{H}, \mathrm{J}=1,0 \quad \mathrm{~Hz}, \mathrm{~J}=8,0 \quad \mathrm{~Hz}), \quad 7,80 \quad(\mathrm{~d}, 1 \mathrm{H}, \mathrm{J}=7,2 \quad \mathrm{~Hz}), 7,56 \quad(\mathrm{~m}, 1 \mathrm{H}), 2,71(\mathrm{~m}, 1 \mathrm{H}), 1,24(\mathrm{~d}, 6 \mathrm{H})$. Anal. $\left(\mathrm{C}_{13} \mathrm{H}_{13} \mathrm{NO}_{4}\right) \mathrm{C}, \mathrm{H}, \mathrm{N}$

6-Isopropoxyquinoline-4-one-3-carboxylic acid 19

Mp 299-300 ${ }^{\circ} \mathrm{C}:{ }^{1} \mathrm{H}$ NMR (DMSO-d 6 ) $\delta$ 15,38 (brs,1H), 13,11 (brs,1H), 8,70 (s,1H), 8,20 $\left(\begin{array}{lllllll}(\mathrm{dd}, 1 \mathrm{H}, \mathrm{J}=1,2 & \mathrm{Hz}, \mathrm{J}=8,4 & \mathrm{~Hz}\end{array}\right), \quad 7,85 \quad(\mathrm{~d}, 1 \mathrm{H}, \mathrm{J}=7,8 \quad \mathrm{~Hz}), 7,59 \quad(\mathrm{dd}, 1 \mathrm{H}, \quad \mathrm{J}=7,8 \quad \mathrm{~Hz}, \quad \mathrm{~J}=8,4$ $\mathrm{Hz}), 3,71(\mathrm{~m}, 1 \mathrm{H}), 1,34(\mathrm{~d}, 6 \mathrm{H})$. Anal. $\left(\mathrm{C}_{13} \mathrm{H}_{13} \mathrm{NO}_{4}\right) \mathrm{C}, \mathrm{H}, \mathrm{N}$

8-Methoxyquinoline-4-one-3-carboxylic acid $\mathbf{2 0}$

Mp 278-280 ${ }^{\circ} \mathrm{C}:{ }^{1} \mathrm{H}$ NMR (DMSO-d $\left.{ }_{6}\right) \delta 15,31$ (brs,1H), 13,03 (brs, 1H), 8,60 (d,1H,J=6,8Hz), 7,85 $(\mathrm{dd}, 1 \mathrm{H}, \mathrm{J}=8,1 \mathrm{~Hz}, \mathrm{~J}=1,6 \mathrm{~Hz}), 7,54(\mathrm{~m}, 2 \mathrm{H}), 4,08(\mathrm{~s}, 3 \mathrm{H})$. Anal. $\left(\mathrm{C}_{11} \mathrm{H}_{9} \mathrm{NO}_{4}\right) \mathrm{C}, \mathrm{H}, \mathrm{N}$

6-Phenoxyquinoline-4-one-3-carboxylic acid 21

Mp 267-269 ${ }^{\circ} \mathrm{C}:{ }^{1} \mathrm{H}$ NMR (DMSO-d 6 ) $\delta 15,26$ (brs, $\left.1 \mathrm{H}\right), 13,85$ (brs, $\left.1 \mathrm{H}\right), 8,84(\mathrm{~s}, 1 \mathrm{H}), 7,96(\mathrm{~d}, 1 \mathrm{H}, 9,0$ $\mathrm{Hz}), 7,70(\mathrm{dd}, 1 \mathrm{H}, \mathrm{J}=9,0 \mathrm{~Hz}, \mathrm{~J}=2,8 \mathrm{~Hz}), 7,57(\mathrm{~d}, 1 \mathrm{H}, \mathrm{J}=2,8 \mathrm{~Hz}), 7,49(\mathrm{~m}, 2 \mathrm{H}), 7,27(\mathrm{~m}, 1 \mathrm{H}), 7,16(\mathrm{~d}, 2 \mathrm{H})$. Anal. $\left(\mathrm{C}_{16} \mathrm{H}_{11} \mathrm{NO}_{4}\right) \mathrm{C}, \mathrm{H}, \mathrm{N}$

6-Butylquinoline-4-one-3-carboxylic acid 22

Mp 235-236 ${ }^{\circ} \mathrm{C}:{ }^{1} \mathrm{H}$ NMR (DMSO-d 6 ) $\delta$ 15,46 (brs,1H), 13,42 (brd,1H), 8,87 (d,1H,J=6,2 Hz), 8,09 $(\mathrm{s}, 1 \mathrm{H}), 7,76(\mathrm{~m}, 2 \mathrm{H}), 2,78(\mathrm{t}, 2 \mathrm{H}), 1,63(\mathrm{~m}, 2 \mathrm{H}), 1,33(\mathrm{~m}, 2 \mathrm{H}), 0,93(\mathrm{t}, 3 \mathrm{H})$. Anal. $\left(\mathrm{C}_{14} \mathrm{H}_{15} \mathrm{NO}_{3}\right) \mathrm{C}, \mathrm{H}, \mathrm{N}$

8-Ethylquinoline-4-one-3-carboxylic acid 23

Mp 278,5-279, $5^{\circ} \mathrm{C}:{ }^{1} \mathrm{H}$ NMR (DMSO-d 6 ) $\delta$ 15,31 (brs, $\left.1 \mathrm{H}\right), 12,91$ (brs, $\left.1 \mathrm{H}\right), 8,65$ (s,1H), 8,17 $(\mathrm{dd}, 1 \mathrm{H}, \mathrm{J}=7,8 \mathrm{~Hz}, \mathrm{~J}=0,9 \mathrm{~Hz}), 7,75(\mathrm{~d}, 1 \mathrm{H}, \mathrm{J}=7,2 \mathrm{~Hz}), 7,53(\mathrm{t}, 1 \mathrm{H}, \mathrm{J}=7,8 \mathrm{~Hz}), 3,02(\mathrm{q}, 2 \mathrm{H}), 1,28(\mathrm{t}, 3 \mathrm{H})$. Anal. $\left(\mathrm{C}_{12} \mathrm{H}_{11} \mathrm{NO}_{3}\right) \mathrm{C}, \mathrm{H}, \mathrm{N}$

6-Propoxyquinoline-4-one-3-carboxylic acid 24 
Mp 265-267 ${ }^{\circ} \mathrm{C}:{ }^{1} \mathrm{H}$ NMR (DMSO-d $\left.{ }_{6}\right) \delta$ 15,58 (brs,1H), 13,47 (brs, $\left.1 \mathrm{H}\right), 8,82$ (d,1H,J=6,2Hz), 7,79 $(\mathrm{d}, 1 \mathrm{H}, \mathrm{J}=9 \mathrm{~Hz}), 7,63(\mathrm{~d}, 1 \mathrm{H}, \mathrm{J}=2,8 \mathrm{~Hz}), 7,53(\mathrm{dd}, 1 \mathrm{H}, \mathrm{J}=9 \mathrm{~Hz}, \mathrm{~J}=2,8 \mathrm{~Hz}), 4,09(\mathrm{t}, 2 \mathrm{H}), 1,81(\mathrm{~m}, 2 \mathrm{H}), 1,03(\mathrm{t}, 3 \mathrm{H})$. Anal. $\left(\mathrm{C}_{13} \mathrm{H}_{13} \mathrm{NO}_{4}\right) \mathrm{C}, \mathrm{H}, \mathrm{N}$

7-Trifluoromethylquinoline-4-one-3-carboxylic acid 25

Mp 249-250 ${ }^{\circ} \mathrm{C}:{ }^{1} \mathrm{H}$ NMR (DMSO-d 6 ) $\delta$ 14,89 (brs,1H), 13,62 (brs,1H), 9,05 (s,1H), 8,47 $(\mathrm{d}, 1 \mathrm{H}, \mathrm{J}=8,4 \mathrm{~Hz}), 8,19(\mathrm{~s}, 1 \mathrm{H}), 7,88(\mathrm{dd}, 1 \mathrm{H}, \mathrm{J}=1,6 \mathrm{~Hz}, \mathrm{~J}=8,4 \mathrm{~Hz})$. Anal. $\left(\mathrm{C}_{11} \mathrm{H}_{6} \mathrm{~F}_{3} \mathrm{NO}_{3}\right) \mathrm{C}, \mathrm{H}, \mathrm{N}$

6-Bromoquinoline-4-one-3-carboxylic acid 26

Mp 276-278 ${ }^{\circ} \mathrm{C}:{ }^{1} \mathrm{H}$ NMR (DMSO-d $\left.{ }_{6}\right) \quad \delta \quad 14,96$ (brs,1H), 13,51 (brs,1H), 8,93 (s,1H), 8,35 $(\mathrm{d}, 1 \mathrm{H}, \mathrm{J}=2,2 \mathrm{~Hz}), 8,03(\mathrm{dd}, 1 \mathrm{H}, \mathrm{J}=2,2 \mathrm{~Hz}, \mathrm{~J}=9,0 \mathrm{~Hz}), 7,78(\mathrm{~d}, 1 \mathrm{H}, \mathrm{J}=9,0 \mathrm{~Hz})$. Anal. $\left(\mathrm{C}_{10} \mathrm{H}_{6} \mathrm{BrNO}_{3}\right) \mathrm{C}, \mathrm{H}, \mathrm{N}, \mathrm{Br}$

6-Chloroquinoline-4-one-3-carboxylic acid 27

Mp 277-278 ${ }^{\circ} \mathrm{C}:{ }^{1} \mathrm{H}$ NMR (DMSO-d 6 ) $\delta$ 15,03 (brs,1H), 13,62 (brs,1H), 8,96 (s,1H), 8,23 $(\mathrm{d}, 1 \mathrm{H}, \mathrm{J}=2,2 \mathrm{~Hz}), 7,96(\mathrm{dd}, 1 \mathrm{H}, \mathrm{J}=9,0 \mathrm{~Hz}, \mathrm{~J}=2,2 \mathrm{~Hz}), 7,88(\mathrm{~d}, 1 \mathrm{H}, \mathrm{J}=9,0 \mathrm{~Hz})$. Anal. $\left(\mathrm{C}_{10} \mathrm{H}_{6} \mathrm{ClNO}_{3}\right) \mathrm{C}, \mathrm{H}, \mathrm{N}, \mathrm{Cl}$

6-Dimethylaminoquinoline-4-one-3-carboxylic acid 28

Mp 258-259 ${ }^{\circ} \mathrm{C}:{ }^{1} \mathrm{H}$ NMR (DMSO-d 6 ) $\delta 15,82$ (brs, 1H), 13,20 (brs, 1H), 8,66 (s,1H), 7,67 (d,1H,J=9,0 $\mathrm{Hz}), 7,47(\mathrm{dd}, 1 \mathrm{H}, \mathrm{J}=9,0 \mathrm{~Hz}, \mathrm{~J}=2,8 \mathrm{~Hz}), 7,26(\mathrm{~d}, 1 \mathrm{H}, \mathrm{J}=2,8 \mathrm{~Hz}), 3,04(\mathrm{~s}, 6 \mathrm{H})$. Anal. $\left(\mathrm{C}_{12} \mathrm{H}_{12} \mathrm{~N}_{2} \mathrm{O}_{3}\right) \mathrm{C}, \mathrm{H}, \mathrm{N}$

8-Methylquinoline-4-one-3-carboxylic acid 29

Mp 271-273 ${ }^{\circ} \mathrm{C}:{ }^{1} \mathrm{H}$ NMR (DMSO-d 6 ) $\delta$ 14,23 (brs,1H), 12,60 (brs,1H), 8,57 (s,1H), 8,10 $(\mathrm{d}, 1 \mathrm{H}, \mathrm{J}=8,4 \mathrm{~Hz}), 7,69(\mathrm{~d}, 1 \mathrm{H}, \mathrm{J}=6,7 \mathrm{~Hz}), 7,45(\mathrm{~m}, 1 \mathrm{H}), 2,45(\mathrm{~s}, 3 \mathrm{H})$. Anal. $\left(\mathrm{C}_{11} \mathrm{H}_{9} \mathrm{NO}_{3}\right) \mathrm{C}, \mathrm{H}, \mathrm{N}$

6-Fluoroquinoline-4-one-3-carboxylic acid 30

Mp 286-287 ${ }^{\circ} \mathrm{C}:{ }^{1} \mathrm{H}$ NMR (DMSO-d 6 ) $\delta$ 15,06 (brs,1H), 13,60 (brs, $\left.1 \mathrm{H}\right), 8,89(\mathrm{~s}, 1 \mathrm{H})$, 7,84 (m,3H). Anal. $\left(\mathrm{C}_{10} \mathrm{H}_{6} \mathrm{FNO}_{3}\right) \mathrm{C}, \mathrm{H}, \mathrm{N}$

8-Fluoroquinoline-4-one-3-carboxylic acid $\mathbf{3 1}$

Mp 275-280 ${ }^{\circ} \mathrm{C}:{ }^{1} \mathrm{H}$ NMR (DMSO-d 6 ) $\delta$ 14,98 (brs,1H), 13,61 (brs,1H), 8,66 (s,1H), 8,10 $(\mathrm{d}, 1 \mathrm{H}, \mathrm{J}=8,4 \mathrm{~Hz}), 7,85(\mathrm{~m}, 1 \mathrm{H}), 7,60(\mathrm{~m}, 1 \mathrm{H})$. Anal. $\left(\mathrm{C}_{10} \mathrm{H}_{6} \mathrm{FNO}_{3}\right) \mathrm{C}, \mathrm{H}, \mathrm{N}$

5,7-Dichloroquinoline-4-one-3-carboxylic acid 32

Mp 296-298 ${ }^{\circ} \mathrm{C}:{ }^{1} \mathrm{H}$ NMR (DMSO-d 6 ) $\delta 14,93$ (brs, $\left.1 \mathrm{H}\right), 13,41$ (brs, $\left.1 \mathrm{H}\right), 8,90(\mathrm{~s}, 1 \mathrm{H}), 7,79$ (d,1H,J=2,2 $\mathrm{Hz}), 7,69(\mathrm{~d}, 1 \mathrm{H}, \mathrm{J}=2,2 \mathrm{~Hz})$. Anal. $\left(\mathrm{C}_{10} \mathrm{H}_{5} \mathrm{Cl}_{2} \mathrm{NO}_{3}\right) \mathrm{C}, \mathrm{H}, \mathrm{N}, \mathrm{Cl}$

6,8-Dimethylquinoline-4-one-3-carboxylic acid 33

Mp 298-300 ${ }^{\circ} \mathrm{C}:{ }^{1} \mathrm{H}$ NMR (DMSO-d 6 ) $\delta 15,36$ (brs, $\left.1 \mathrm{H}\right), 12,75$ (brs, $\left.1 \mathrm{H}\right), 8,59$ (s,1H), 7,90 (s, 1H), 7,53 $(\mathrm{s}, 1 \mathrm{H}), 2,57(\mathrm{~s}, 3 \mathrm{H}), 2,43(\mathrm{~s}, 3 \mathrm{H})$. Anal. $\left(\mathrm{C}_{12} \mathrm{H}_{11} \mathrm{NO}_{3}\right) \mathrm{C}, \mathrm{H}, \mathrm{N}$

7,8-Dimethylquinoline-4-one-3-carboxylic acid 34

Mp 272-273 ${ }^{\circ} \mathrm{C}:{ }^{1} \mathrm{H}$ NMR (DMSO-d $\left.{ }_{6}\right) \delta 15,39$ (brs, $\left.1 \mathrm{H}\right), 12,66$ (brs, $\left.1 \mathrm{H}\right), 8,57(\mathrm{~s}, 1 \mathrm{H}), 8,02(\mathrm{~d}, 1 \mathrm{H}, \mathrm{J}=8,4$ $\mathrm{Hz}), 7,40(\mathrm{~d}, 1 \mathrm{H}, \mathrm{J}=8,4 \mathrm{~Hz}), 2,45(2 \mathrm{~s}, 6 \mathrm{H})$. Anal. $\left(\mathrm{C}_{12} \mathrm{H}_{11} \mathrm{NO}_{3}\right) \mathrm{C}, \mathrm{H}, \mathrm{N}$

8-Bromoquinoline-4-one-3-carboxylic acid 35

Mp 270-271 ${ }^{\circ} \mathrm{C}:{ }^{1} \mathrm{H}$ NMR (DMSO-d $\left.{ }_{6}\right) \delta 14,88$ (brs, $\left.1 \mathrm{H}\right), 12,78$ (brs, $\left.1 \mathrm{H}\right), 8,71(\mathrm{~s}, 1 \mathrm{H}), 8,24(\mathrm{~d}, 1 \mathrm{H}, \mathrm{J}=8,1$ $\mathrm{Hz}), 8,21(\mathrm{~d}, 1 \mathrm{H}, \mathrm{J}=7,8 \mathrm{~Hz}), 7,51(\mathrm{dd}, 1 \mathrm{H}, \mathrm{J}=8,1 \mathrm{~Hz}, \mathrm{~J}=7,8 \mathrm{~Hz})$. Anal. (C10H6BrNO3) C,H,N,Br

8-Chloroquinoline-4-one-3-carboxylic acid 36

Mp 268-270 ${ }^{\circ}:{ }^{1} \mathrm{H}$ NMR (DMSO-d $)_{6} \delta$ 12,91 (brs,1H), 8,63 (s,1H), 8,25 (d,1H,J=8,1Hz), 8,06 $(\mathrm{d}, 1 \mathrm{H}, \mathrm{J}=8,1 \mathrm{~Hz}), 7,59(\mathrm{t}, 1 \mathrm{H}, \mathrm{J}=8,1 \mathrm{~Hz})$. Anal. $\left(\mathrm{C}_{10} \mathrm{H}_{6} \mathrm{ClNO}_{3}\right) \mathrm{C}, \mathrm{H}, \mathrm{N}, \mathrm{Cl}$ 
5,8-Dimethylquinoline-4-one-3-carboxylic acid 37

Mp 252-253 ${ }^{\circ} \mathrm{C}:{ }^{1} \mathrm{H}$ NMR (DMSO-d 6 ) $\delta$ 12,49 (brs, $\left.1 \mathrm{H}\right), 8,59(\mathrm{~s}, 1 \mathrm{H}), 7,57(\mathrm{~d}, 1 \mathrm{H}, \mathrm{J}=7,5 \mathrm{~Hz}), 7,22$ $(\mathrm{d}, 1 \mathrm{H}, \mathrm{J}=7,5 \mathrm{~Hz}), 2,83(\mathrm{~s}, 3 \mathrm{H}), 2,53(\mathrm{~s}, 3 \mathrm{H})$. Anal. $\left(\mathrm{C}_{12} \mathrm{H}_{11} \mathrm{NO}_{3}\right) \mathrm{C}, \mathrm{H}, \mathrm{N}$

8-Methyl-7-chloroquinoline-4-one-3-carboxylic acid $\mathbf{3 8}$

Mp 279-281 ${ }^{\circ} \mathrm{C}:{ }^{1} \mathrm{H} \quad \mathrm{NMR} \quad\left(\mathrm{DMSO}_{-} \mathrm{d}_{6}\right) \quad \delta \quad 14,94 \quad$ (brs,1H), 12,69 (brs, $\left.1 \mathrm{H}\right), 8,57 \quad(\mathrm{~s}, 1 \mathrm{H}), 8,06$ $(\mathrm{d}, 1 \mathrm{H}, \mathrm{J}=8,7 \mathrm{~Hz}), 7,57(\mathrm{~d}, 1 \mathrm{H}, \mathrm{J}=8,7 \mathrm{~Hz}), 2,57(\mathrm{~s}, 3 \mathrm{H})$. Anal. $\left(\mathrm{C}_{11} \mathrm{H}_{8} \mathrm{ClNO}_{3}\right) \mathrm{C}, \mathrm{H}, \mathrm{N}, \mathrm{Cl}$

7,8-Dichloroquinoline-4-one-3-carboxylic acid 39

Mp 270-272 ${ }^{\circ} \mathrm{C}:{ }^{1} \mathrm{H} \quad \mathrm{NMR} \quad\left(\mathrm{DMSO}-\mathrm{d}_{6}\right.$ ) $\delta \quad 14,69$ (brs,1H), $12,96 \quad$ (brs, $\left.1 \mathrm{H}\right), 8,62 \quad(\mathrm{~s}, 1 \mathrm{H}), \quad 8,21$ $(\mathrm{d}, 1 \mathrm{H}, \mathrm{J}=9,0 \mathrm{~Hz}), 7,79(\mathrm{~d}, 1 \mathrm{H}, \mathrm{J}=9,0 \mathrm{~Hz})$. Anal. $\left(\mathrm{C}_{10} \mathrm{H}_{5} \mathrm{Cl}_{2} \mathrm{NO}_{3}\right) \mathrm{C}, \mathrm{H}, \mathrm{N}, \mathrm{Cl}$

1-Methylquinoline-4-one-3-carboxylic acid 40

Mp 255-257 ${ }^{\circ} \mathrm{C}:{ }^{1} \mathrm{H}$ NMR (DMSO-d $\left.\mathrm{d}_{6}\right) \delta 15,13$ (brs,1H), 9,00 (s,1H), 8,39 (d,1H,J=7,8 Hz), 7,96 $(\mathrm{m}, 2 \mathrm{H}), 7,68(\mathrm{~m}, 1 \mathrm{H}), 4,11(\mathrm{~s}, 3 \mathrm{H})$. Anal. $\left(\mathrm{C}_{11} \mathrm{H}_{9} \mathrm{NO}_{3}\right) \mathrm{C}, \mathrm{H}, \mathrm{N}$

1,6,8-Trimethylquinoline-4-one-3-carboxylic acid 41

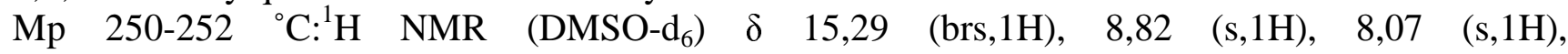
7,59(s,1H),4,32(s,3H),2,86(s,3H),2,43(s,3H). Anal. $\left(\mathrm{C}_{13} \mathrm{H}_{13} \mathrm{NO}_{3}\right) \mathrm{C}, \mathrm{H}, \mathrm{N}$

1,6-Dimethylquinoline-4-one-3-carboxylic acid $\mathbf{4 2}$

Mp 260-262 ${ }^{\circ} \mathrm{C}:{ }^{1} \mathrm{H}$ NMR (DMSO-d $\left.{ }_{6}\right) \delta 15,22(\mathrm{brs}, 1 \mathrm{H}), 8,92(\mathrm{~s}, 1 \mathrm{H}), 8,14(\mathrm{~s}, 1 \mathrm{H}), 7,84(\mathrm{~d}, 1 \mathrm{H}, \mathrm{J}=9,0$ $\mathrm{Hz}), 7,77(\mathrm{~d}, 1 \mathrm{H}, \mathrm{J}=9,0 \mathrm{~Hz}), 4,07(\mathrm{~s}, 3 \mathrm{H}), 2,50(\mathrm{~s}, 3 \mathrm{H})$. Anal. $\left(\mathrm{C}_{12} \mathrm{H}_{11} \mathrm{NO}_{3}\right) \mathrm{C}, \mathrm{H}, \mathrm{N}$ 
Table of elemental analyses for compounds 1-42.

\begin{tabular}{|c|c|c|c|c|c|c|c|c|}
\hline $\begin{array}{l}\text { № of } \\
\text { compoun } \\
\text { d }\end{array}$ & $\begin{array}{l}\text { C\%,calc } \\
\text { ulated }\end{array}$ & $\begin{array}{l}\mathrm{H} \% \text {, } \\
\text { calculate } \\
\text { d }\end{array}$ & $\begin{array}{l}\mathrm{N} \% \text {, } \\
\text { calculate } \\
\mathrm{d}\end{array}$ & $\begin{array}{l}\mathrm{Cl} \% \text {, } \\
\text { calculate } \\
\mathrm{d}\end{array}$ & $\begin{array}{l}\mathrm{C} \% \text {,found } \\
\text { ed }\end{array}$ & $\begin{array}{l}\mathrm{H} \% \text {, } \\
\text { founde } \\
\mathrm{d}\end{array}$ & $\begin{array}{l}\mathrm{N} \% \text {, } \\
\text { founde } \\
\mathrm{d}\end{array}$ & $\begin{array}{l}\mathrm{Cl} \% \text {, } \\
\text { founde } \\
\mathrm{d}\end{array}$ \\
\hline 1 & 38,12 & 1,92 & 4,45 & - & 37,98 & 1,96 & 4,50 & \\
\hline 2 & 56,66 & 3,03 & 6,01 & - & 56,90 & 2,97 & 5,92 & \\
\hline 3 & 56,66 & 3,03 & 6,01 & - & 56,53 & 3,00 & 6,05 & \\
\hline 4 & 56,66 & 3,03 & 6,01 & - & 56,45 & 3,09 & 6,09 & \\
\hline 5 & 58,30 & 3,67 & 5,67 & - & 58,45 & 3,60 & 5,52 & \\
\hline 6 & 46,54 & 1,95 & 5,43 & 27,48 & 46,33 & 2,01 & 5,51 & 27,59 \\
\hline 7 & 41,06 & 1,38 & 4,79 & 36,36 & 41,30 & 1,40 & 4,70 & 36,42 \\
\hline 8 & 55,52 & 3,23 & 4,98 & - & 55,69 & 3,30 & 4,89 & \\
\hline 9 & 70,29 & 3,79 & 5,85 & - & 70,00 & 3,85 & 5,91 & \\
\hline 10 & 61,80 & 4,75 & 6,01 & - & 61,66 & 4,71 & 6,10 & \\
\hline 11 & 60,28 & 4,14 & 6,39 & - & 60,37 & 4,00 & 6,30 & \\
\hline 12 & 63,49 & 3,73 & 7,40 & - & 63,24 & 3,80 & 7,47 & \\
\hline 13 & 61,08 & 4,75 & 6,01 & - & 61,19 & 4,79 & 5,93 & \\
\hline 14 & 59,83 & 5,58 & 11,63 & - & 59,70 & 5,50 & 11,79 & \\
\hline 15 & 65,02 & 4,46 & 6,89 & - & 65,27 & 4,50 & 6,80 & \\
\hline 16 & 62,34 & 3,92 & 6,06 & - & 62,45 & 3,88 & 5,98 & \\
\hline 17 & 57,83 & 4,45 & 5,62 & - & 57,70 & 4,50 & 5,50 & \\
\hline 18 & 67,52 & 5,67 & 6,06 & - & 67,45 & 5,59 & 6,13 & \\
\hline 19 & 63,15 & 5,30 & 5,66 & - & 63,00 & 5,21 & 5,80 & \\
\hline 20 & 60,28 & 4,14 & 6,39 & - & 60,41 & 4,22 & 6,29 & \\
\hline 21 & 68,33 & 3,94 & 4,98 & - & 68,56 & 3,86 & 4,81 & \\
\hline 22 & 68,56 & 6,16 & 5,71 & - & 68,31 & 6,05 & 5,60 & \\
\hline 23 & 66,35 & 5,10 & 6,45 & - & 66,08 & 5,09 & 6,59 & \\
\hline 24 & 63,15 & 5,30 & 5,66 & - & 63,02 & 5,33 & 5,80 & \\
\hline 25 & 51,38 & 2,35 & 5,45 & - & 51,26 & 2,39 & 5,50 & \\
\hline 26 & 44,81 & 2,26 & 5,23 & - & 44,95 & 2,30 & 5,18 & \\
\hline 27 & 53,71 & 2,70 & 6,26 & 15,85 & 53,81 & 2,75 & 6,19 & 15,90 \\
\hline 28 & 62,06 & 5,21 & 12,06 & - & 62,15 & 5,29 & 11,98 & \\
\hline 29 & 65,02 & 4,46 & 6,89 & - & 65,19 & 5,19 & 6,76 & \\
\hline 30 & 57,98 & 2,92 & 6,76 & - & 57,86 & 2,88 & 6,81 & \\
\hline 31 & 57,98 & 2,92 & 6,76 & - & 58,09 & 2,89 & 6,69 & \\
\hline 32 & 46,54 & 1,95 & 5,43 & 27,48 & 46,49 & 1,99 & 5,50 & 27,60 \\
\hline 33 & 66,35 & 5,10 & 6,45 & - & 66,50 & 5,09 & 6,39 & \\
\hline 34 & 66,35 & 5,10 & 6,45 & - & 66,18 & 5,18 & 6,59 & \\
\hline 35 & 44,81 & 2,26 & 5,23 & - & 44,99 & 2,35 & 5,19 & \\
\hline 36 & 53,71 & 2,70 & 6,26 & 15,85 & 53,49 & 2,80 & 6,39 & 15,79 \\
\hline 37 & 66,35 & 5,10 & 6,45 & - & 66,50 & 5,15 & 6,40 & \\
\hline 38 & 55,60 & 3,39 & 5,89 & 14,92 & 55,65 & 3,36 & 5,85 & \\
\hline 39 & 46,54 & 1,95 & 5,43 & 27,48 & 46,70 & 1,99 & 5,38 & 27,44 \\
\hline 40 & 65,02 & 4,46 & 6,89 & - & 65,29 & 4,40 & 6,78 & \\
\hline 41 & 67,52 & 5,67 & 6,06 & - & 67,39 & 5,77 & 6,19 & \\
\hline 42 & 66,35 & 5,10 & 6,45 & - & 66,00 & 5,19 & 6,61 & \\
\hline
\end{tabular}

